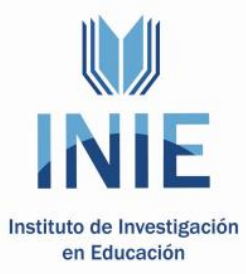

Actualidades Investigativas en Educación

Revista Electrónica publicada por el

Instituto de Investigación en Educación

Universidad de Costa Rica

ISSN 1409-4703

http://revista.inie.ucr.ac.cr

COSTA RICA
REVISTA

Actualidades

Investigativas

en Educación

\title{
LA COMUNICACIÓN NO VERBAL-EXPRESIVO CORPORAL EN UN CONTEXTO ESCOLAR INTERCULTURAL EN EL NORTE DE ÁFRICA: ESTUDIO COMPARATIVO ENTRE NIÑOS EUROPEOS VS AMAZIGH NONVERBAL -CORPORAL EXPRESSIVE COMMUNICATION IN AN INTERCULTURAL SCHOOL CONTEXT IN NORTH AFRICA: COMPARATIVE STUDY BETWEEN EUROPEAN VS AMAZIGH CHILDREN
}

\author{
Volumen 10, Número 1 \\ pp. $1-18$
}

Este número se publicó el 30 de abril de 2010

\author{
Clemente Rodríguez \\ Manuel Ortega \\ José Álvarez \\ Oswaldo Lorenzo \\ Roberto Cremades
}

La revista está indexada en los directorios:

LATINDEX, REDALYC, IRESIE, CLASE, DIALNET, DOAJ, E-REVIST@S,

La revista está incluida en los sitios:

REDIE, RINACE, OEI, MAESTROTECA, PREAL, HUASCARAN, CLASCO

Los contenidos de este artículo están bajo una licencia Creative Commons






\title{
LA COMUNICACIÓN NO VERBAL-EXPRESIVO CORPORAL EN UN CONTEXTO ESCOLAR INTERCULTURAL EN EL NORTE DE ÁFRICA: ESTUDIO COMPARATIVO ENTRE NIÑOS EUROPEOS VS AMAZIGH NONVERBAL -CORPORAL EXPRESSIVE COMMUNICATION IN AN INTERCULTURAL SCHOOL CONTEXT IN NORTH AFRICA: COMPARATIVE STUDY BETWEEN EUROPEAN VS AMAZIGH CHILDREN
}

\author{
Clemente Rodríguez ${ }^{1}$ \\ Manuel Ortega ${ }^{2}$ \\ José Álvarez ${ }^{3}$ \\ Oswaldo Lorenzo 4 \\ Roberto Cremades 5
}

\begin{abstract}
Resumen: En este artículo se ha llevado a cabo un estudio comparativo acerca de los diferentes tipos de comportamiento relacionados con la comunicación no verbal y la expresión corporal que se dan entre niños de edad escolar con pertenencia a dos grupos étnicos diferentes (europeo y amazigh). El estudio se ha desarrollado en la ciudad de Melilla (España), población situada en el norte occidental de África y caracterizada por una gran diversidad social y cultural de sus habitantes (europeos, imazighen 6 , judíos sefardíes e hindúes). Para ello, han participado 100 alumnos (57 niños y 43 niñas), siendo el 50\% de origen cultural europeo y el $50 \%$ de origen cultural amazigh, a los que se evaluó a través de diferentes pruebas de carácter observacional. Los resultados obtenidos revelan que los niños europeos usan más la comunicación no verbal que los de la cultura amazigh.
\end{abstract}

Palabras Clave: COMUNICACIÓN NO VERBAL, EXPRESIÓN CORPORAL, DIVERSIDAD CULTURAL, TECNOLOGÍAS DE LA INFORMACIÓN Y LA COMUNICACIÓN (TIC).

Abstract: This article has conducted a comparative study about different kinds of behaviour related to non verbal communication and the corporal expression that happen among school-age children who belong to two different ethnic groups (European and Amazigh). The study has been developed in the city of Melilla (Spain), located in western North Africa. This city is characterized by social and cultural diversity of its citizens (Europeans, Berbers, Sephardic Jews and Indians). Some 100 students participated (57 boys and 43 gilrs), of which $50 \%$ were of European cultural origin and 50\% of Amazigh cultural origin, which was evaluated through different observational tests. Results show that European children use nonverbal communication more than those of the Amazigh culture. Keywords: NONVERBAL COMMUNICATION, CORPORAL EXPRESSION, CULTURAL DIVERSITY, INFORMATION AND COMMUNICATION TECHNOLOGIES (ICT).

\footnotetext{
${ }^{1}$ Doctor en Pedagogía. Profesor de la Universidad de Granada (España). Dirección electrónica: clerosa@ugr.es

2 Doctor en Ciencias de la Educación. Profesor de la Universidad de Granada (España). Dirección electrónica: manorca@ugr.es

${ }^{3}$ Doctor en Pedagogía. Profesor de la Universidad de Granada (España). Dirección electrónica: alvarez@ugr.es

${ }^{4}$ Doctor en Ciencias de la Educación por la Universidad Nacional de Educación a Distancia. Profesor de la Universidad de Granada (España).Dirección electrónica: oswaldo@ugr.es

5 Doctor por la Universidad de Granada con mención de Doctor Europeus. Profesor de la Universidad de Granada (España). Dirección electrónica: fusas@ugr.es. Persona de contacto
}

Artículo recibido: 13 de enero, 2010

Aprobado: 12 de abril, 2010

${ }^{6}$ Plural de amazigh. A lo largo del trabajo se usará uno u otro término: amazigh o bereber, aunque se trate de etnias no exactamente similares. 


\section{Introducción}

Entre los trabajos clásicos sobre categorización del comportamiento humano en sus diferentes facetas (Warren, 1980) destaca el de Ekman y Friesen (1969), que mantiene aún cierto grado de actualidad e intenta establecer posibles fuentes primordiales del comportamiento no verbal. En este estudio se apuntan tres bases como determinantes de la conducta no verbal en los seres humanos:

1.Problemas neurológicos heredados.

2. Experiencias comunes a todos los miembros del grupo social.

3. Experiencias variables en función de la cultura del grupo.

El trabajo de Ekman y Friesen se ve pronto complementado con la perspectiva de EiblEibesfeldt (1970), quien sugiere que es posible también encontrar secuencias de comportamiento no verbal que manifiesten semejanzas transculturales, asumiendo que efectivamente las distintas culturas se encargarán de diferenciarse entre sí en el nivel no verbal, influidas por características tanto personales como ambientales.

Más recientemente, es posible encontrar distintos estudios sobre antropología y psicología social y cultural que abordan, desde diferentes aproximaciones, la comunicación no verbal y sus manifestaciones asociadas de expresión y conducta corporal en contextos interculturales, humanos y escolares (Elfenbein y Ambady, 2002; Hendry, 1999; Marsh, Elfenbein, y Ambady, 2003; Monaghan y Goodman, 2007; Shiraev y Levy, 2006).

De este modo, las tradicionales fórmulas de saludo amistoso, resultantes de la combinación de expresiones verbal-no verbal, que se dan en todos los pueblos del mundo, por ejemplo, el movimiento de cabeza de los árabes e israelíes acompañado en cada caso de las palabras Sallan allec y Shalom, Shalom, lo que para los dos significa paz, está hoy evolucionando por el aumento de la convivencia e intercambio cotidiano entre culturas y costumbres, dejando progresivamente de ser características individualizadas de los pueblos para convertirse cada vez más en adaptativas y menos diferenciadoras.

Entre las distintas modalidades de expresión no verbal son quizá las expresiones faciales primarias las que han sido más estudiadas, posiblemente porque estas se advierten fácilmente tanto en primates no humanos como en culturas letradas y ágrafas de todo el Volumen 10, Número 1, Año 2010, ISSN 1409-4703 
planeta (Vick, Waller, Parr, Smith y Bard, 2007). Así, Marsh, Adams y Kleck (2005) defienden que sería difícil negar en este tipo de expresiones la existencia de un componente genético transmitido de una generación a otra en la especie humana, lo que evidenciaría una asociación universal entre particulares zonas musculares faciales para la expresión de emociones, algo que no implica que la cara de las personas deba ser una especie de caja cerrada en la que cada movimiento o gesto sólo deba expresar algo muy determinado.

\section{La comunicación no verbal en el ámbito escolar}

En el proceso escolar de enseñanza-aprendizaje se establece siempre un clima de interrelación entre educación y lenguaje corporal-no verbal de innegable interés para el profesor y para el desarrollo educativo de los alumnos. En este escenario es destacable la influencia cinética y recíproca de cada individuo en su entorno personal inmediato, influencia no siempre consciente que debería proyectarse en el ámbito educativo conforme a una secuencia deseable de desarrollo de la interacción no verbal entre docentes y discentes (ver figura 1).

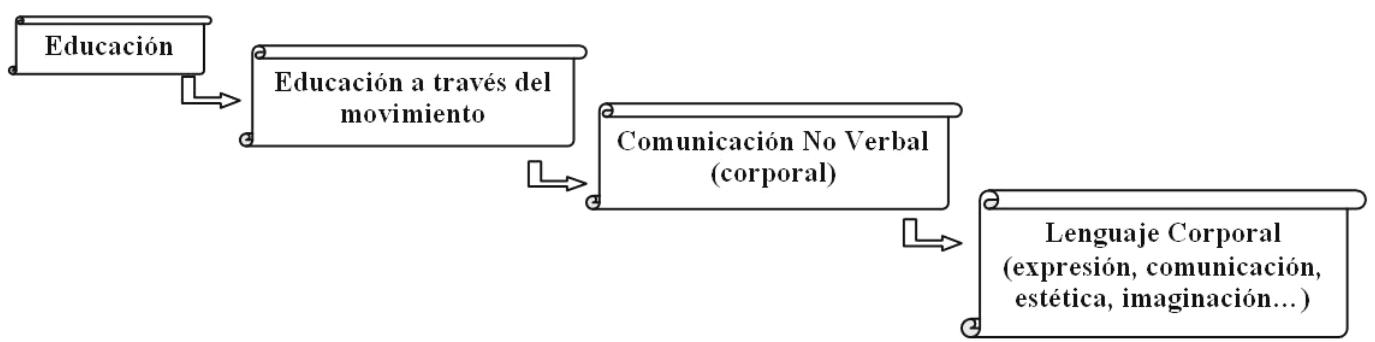

Figura 1: Fases deseables de la interacción no verbal en la escuela.

Long (1998) subraya la importancia de los aspectos no verbales en las etapas iniciales de educación del alumnado, así como de los factores que afectan a la comunicación no verbal en el proceso educativo. Todo ello debe suponer una ayuda para conformar progresivamente las bases de una comunicación no verbal de comprensión "universal", con el fin de que todos los seres humanos sean capaces de percibir correctamente la información no verbal proveniente de sus semejantes y logren codificar y descodificar acertadamente sus expresiones, tan importantes éstas para la mejora de las relaciones interpersonales entre alumnos y profesores y, especialmente, para la atención escolar a la creciente diversidad cultural del alumnado. 
En esta línea de estudio sobre la comunicación no verbal en las aulas, los trabajos de Potoker (1993) y Guo (1999) ponen de manifiesto las diferencias que surgen entre profesores y alumnos de diferentes etnias en su comunicación verbal y, sobre todo, no verbal. Los discentes emigrados e inmersos en un nuevo contexto con orígenes heterogéneos entre el alumnado se enfrentan con frecuencia a situaciones que les crean problemas de desinformación continua, de adaptación y de asimilación de contenidos. Para abordar este problema, se han puesto en práctica iniciativas y experiencias donde se instruye al profesorado en el reconocimiento de expresiones no verbales de niños culturalmente diferentes al alumnado autóctono. Por ejemplo, con este objetivo, Nichol y Watson (2000) realizaron una autoevaluación de la comunicación no verbal de los profesores en su etapa inicial, a través de un recurso denominado "videoturing", alcanzando, posteriormente, estos profesores una mejor relación con sus alumnos no autóctonos.

Por otra parte, Capper (2000) propone que la comunicación no verbal se incluya dentro del programa de lenguas extranjeras y se enseñe de forma intencionada para llegar a conocer mejor la lengua y la cultura de otros países que poseen un repertorio kinésico, proxémico y paralingüístico diferente al de los centros escolares de recepción de alumnos extranjeros.

Partiendo de la revisión teórica hasta aquí expuesta sobre el objeto de estudio de este trabajo, se plantearon los siguientes objetivos:

1. Indagar acerca de los diferentes modos de comunicación no verbal y expresión corporal presentes en alumnos de $5^{\circ}$ curso de educación primaria con origen cultural distinto: europeo y bereber (norteafricano).

2. Estudiar la frecuencia de uso de los modos de comunicación no verbal y expresión corporal empleados por el alumnado de uno y otro grupo étnico.

3. Establecer principios de carácter educativo que puedan ser útiles en el trabajo del profesorado en contextos multiculturales similares al estudiado en este trabajo.

\section{Contextualización y planteamiento del problema}

La presente investigación se ha desarrollado en la Ciudad Autónoma de Melilla, ciudad con aproximadamente 75.000 habitantes censados, situada en el norte de África y en la que conviven varias culturas, principalmente la europea y amazigh y, en menor medida, judía e hindú. Si bien Melilla, pertenece geográficamente al continente africano, su soberanía y Volumen 10, Número 1, Año 2010, ISSN 1409-4703 
administración política corresponden al reino de España, lo que origina una particular situación de mezcolanza multi e intercultural (López-Guzmán, González, Herrera y Lorenzo, 2007), que hace de esta ciudad un escenario muy fértil para llevar a cabo trabajos de naturaleza comparativa entre características y comportamientos culturales de los diferentes grupos humanos ubicados en ella.

La pluralidad étnica de Melilla supone, por tanto, un excelente soporte de diversidad para dar respuesta a interrogantes de investigación en el complejo campo de la comunicación no verbal-expresivo corporal del alumnado. Así, en este contexto el presente trabajo plantea preguntas como las siguientes: ¿existen diferencias de rasgos comunicativos no verbales entre diferentes culturas (bereber y de origen europeo en este caso) que no estén percibiendo docentes y discentes?; ¿quiénes se expresan con mayor frecuencia corporalmente, los alumnos bereberes o los de origen europeo?

\section{Hipótesis de investigación}

\subsection{Hipótesis general}

Existen potenciales diferencias en el plano comunicativo no verbal y de expresión corporal en situaciones escolares entre alumnos de origen europeo y de origen bereber (amazigh).

\subsection{Hipótesis específica}

Existen diferencias estadísticamente significativas entre niños europeos y bereberes en situaciones escolares y en relación con cuatro aspectos de la comunicación no verbal determinados: expresión facial, cambio postural, proxemia y contacto personal.

\section{Método}

El diseño metodológico empleado en este estudio es de naturaleza explícitamente comparativo-causal. El objetivo principal de dichos estudios es explicar relaciones de causalidad comparando grupos de datos sin que la variable independiente (en nuestro caso tipo de etnia de los escolares: europea vs amazigh) que el investigador estudia como posible causa de los cambios observables en el criterio o variable dependiente (en nuestro caso 
comunicación no verbal-expresivo corporal) pueda manipularse, sino acaso seleccionarse (estudio ex post facto). En este tipo de estudios, no estrictamente experimentales, se asume, por tanto, el riesgo implícito de la influencia que pudieran adquirir otras variables potencialmente influyentes, como la condición social de procedencia del alumnado, su género... El diseño metodológico representarse gráficamente de la siguiente forma (ver tabla 1):

Tabla 1

Diagrama del diseño metodológico empleado en la investigación.

\begin{tabular}{|c|c|c|c|}
\hline GRUPOS & $\mathbf{N}$ & $\begin{array}{c}\text { VARIABLE } \\
\text { INDEPENDIENTE } \\
\text { (DOS NIVELES) }\end{array}$ & $\begin{array}{c}\text { VARIABLES } \\
\text { DEPENDIENTES }\end{array}$ \\
\hline $\begin{array}{l}\text { Grupo clase } \\
\text { Grupo clase }\end{array}$ & $\begin{array}{l}50 \\
50\end{array}$ & $\begin{array}{c}\text { Tipo de etnia Europea } \\
\text { Tipo de etnia } \\
\text { Amazigh }\end{array}$ & $\begin{array}{l}\text { CEF, CPO, PRO, CCO } \\
\text { CEF, CPO, PRO, CCO }\end{array}$ \\
\hline
\end{tabular}

Las cuatro variables dependientes contempladas en la tabla 1 corresponden a diversos aspectos de la comunicación no verbal-expresivo corporal, los cuales aparecen definidos a continuación:

- CEF. Cambios de Expresión Facial: cambiar o alterar la condición o apariencia física de la cara.

- CPO. Cambio Postural: cambiar o alterar la condición o apariencia física del cuerpo, a excepción de la cara.

- PRO. Proxemia: acercamiento con intención de establecer relación y comunicación personal entre sujetos.

- CCO. Contacto Corporal: acto y efecto de tocar alguna parte del cuerpo de otra persona.

\subsection{Participantes}

Los estudiantes que han participado en este trabajo fueron seleccionados atendiendo a un muestreo con dos etapas. En primer lugar, se seleccionaron dos centros educativos (colegios) públicos de la ciudad de Melilla, mediante un procedimiento aleatorio, entre los existentes con mayoría de alumnos europeos y mayoría de alumnos bereberes. Una vez 
seleccionados los dos colegios, la segunda etapa consistió en elegir de manera deliberada dos clases de $5^{\circ}$ curso de educación primaria en cada uno de los dos colegios teniendo en cuenta que los grupos tenían que estar conformados por niños de etnia europea exclusivamente (colegio1), aunque también estudiasen en el centro niños de procedencia amazigh y por niños de etnia amazigh exclusivamente (colegio 2), aunque también cursasen sus estudios en el mismos niños de procedencia europea.

Por otra parte todos los alumnos participaron en este estudio con el consentimiento de las autoridades del centro educativo y sus respectivos progenitores. En total, 100 alumnos (57 niños y 43 niñas), con una edad media de 10,5 años y con la siguiente distribución en cada colegio (ver tabla 2):

- Colegio 1: 50 alumnos de cultura europea exclusivamente (25 alumnos y 25 alumnas)

- Colegio 2 : 50 alumnos de cultura amazigh exclusivamente (32 alumnos y 18 alumnas)

Tabla 2

Distribución de alumnos participantes por colegio, curso y sexo.

\begin{tabular}{lcccc}
\hline \multirow{2}{*}{ COLEGIO } & CURSO & \multicolumn{3}{c}{ NÚMERO DE ALUMNOS } \\
\cline { 2 - 5 } & & Niños & Niñas & TOTAL \\
\cline { 2 - 5 } 1 & 5o-A & 14 & 11 & 25 \\
& 5-B & 11 & 14 & 25 \\
2 & 5--A & 15 & 10 & 25 \\
& 5--B & 17 & 8 & 25 \\
& TOTAL & 57 & 43 & 100 \\
\hline
\end{tabular}

\subsection{Instrumentos}

Las distintas pruebas aplicadas a los estudiantes participantes en el estudio han sido las siguientes:

\section{Prueba Observacional de Cambios de Expresión Facial (CEF):}

Contenido de la prueba: visionado de tres cortos cómicos que pueden despertar expresiones y actitudes faciales. Los tres cortos pertenecen al DVD de la película de Disney-Pixar "Monsters, inc.": 
- "For the birds".

- "Mike's New Car".

- "Monsters, inc. (false)".

Técnica empleada: observación Participante. Captura y recogida de imágenes de los cambios de expresión facial de los alumnos mediante cámara de vídeo.

Duración de la prueba: 3 visionados de 4 minutos cada uno (12 minutos en total).

Objetivo de la prueba: evaluar los cambios faciales expresivos en el transcurso del visionado de un mismo film para los dos grupos de alumnos.

\section{Prueba Observacional de Cambio Postural (CPO):}

Contenido de la prueba: visionado de tres cortos cómicos que pueden despertar e inducir cambios posturales transitorios. Los tres cortos pertenecen al DVD de la película de DisneyPixar "Monsters, inc.":

- "For the birds".

- "Mike's New Car".

- "Monsters, inc. (false)".

Técnica empleada: observación Participante. Captura y recogida de imágenes de los cambios posturales de los alumnos mediante cámara de vídeo.

Duración de la prueba: 3 visionados de 4 minutos cada uno (12 minutos en total).

Objetivo de la prueba: evaluar los cambios posturales expresivos en el transcurso del visionado de un mismo film para los dos grupos de alumnos.

\section{Prueba Observacional de Proximidad (PRO)}

Contenido de la prueba: desarrollo de un partido de balón-tiro (un juego de estrategia, desplazamiento y lanzamiento) en el transcurso de una sesión de la asignatura de educación física.

Duración de la prueba: 10 minutos. En el transcurso de la prueba se realizaron 10 interrupciones sistemáticas, una cada 60 segundos, para localizar los diferentes posicionamientos físicos y espaciales de los sujetos. 
Técnica empleada: observación participante. Captura y recogida de imágenes mediante cámara de vídeo de los movimientos y situaciones de proximidad entre los alumnos.

Objetivo de la prueba: evaluar la proximidad interpersonal en el transcurso de una actividad lúdica.

\section{Prueba Observacional de Contacto Corporal (CCO)}

Contenido de la prueba: puesta en marcha del juego espacio-temporal "Cifras y letras" (un juego de estrategia y ubicación espacio-temporal en el que los alumnos deben formar por grupos no preestablecidos siluetas de alguna letra o número) en el transcurso de una sesión de la asignatura de educación física.

Duración de la prueba: 10 minutos. En el transcurso de la prueba se controlan las frecuencias de contactos entre compañeros hasta que queda formada la letra o cifra indicada por el profesor.

Técnica empleada: observación participante. Captura y recogida de imágenes mediante cámara de vídeo de los contactos entre compañeros.

Objetivo de la prueba: evaluar el contacto corporal interpersonal en el transcurso de una actividad lúdica.

\subsection{Recogida de la información y procedimiento}

Para la recogida de información se ha utilizado la observación participante, procedimiento de investigación que implica la interacción entre el investigador y el grupo estudiado y cuyo objetivo es recoger datos de modo sistemático directamente de los contextos y situaciones específicas por las que pasa el grupo (Atkinson y Hammersley, 1998).

De entre las diferentes modalidades posibles de observación participante, en esta investigación se ha empleado la de completo observador, aquella donde hay máxima revelación de la actividad de observación y un alto grado de distanciamiento/no participación del investigador o investigadores. 
En cuanto al procedimiento seguido para la recogida de información, durante los meses de marzo a mayo de 2007 se aplicaron a los alumnos objeto de estudio las cuatro pruebas descritas en el apartado anterior. En el espacio de tres meses todas las pruebas fueron aplicadas en dos ocasiones, durante dos semanas distintas, y de igual modo en cada uno de los grupos de alumnos participantes. Esto ha contribuido a descartar posibles variables extrañas que pudieran haber interferido en los alumnos a la hora de comprender bien la realización de las pruebas.

Tanto en la aplicación, como en el posterior análisis de la información proveniente de las cuatro pruebas de observación sistemática actuaron tres observadores independientes, valorando cada uno por separado los cambios de expresión facial y postural, proximidad interpersonal y contacto corporal. Sólo se tuvieron en cuenta los resultados obtenidos en la segunda aplicación de las pruebas.

\subsection{Criterios de calidad de la recogida de información y el procedimiento}

Las cuatro pruebas utilizadas fueron elaboradas ad hoc, por su naturaleza claramente observacional, contemplando como criterio de calidad la concordancia entre los tres observadores participantes en el estudio. Para la concordancia entre dichos observadores se ha calculado el coeficiente Kappa de Cohen, un estadístico de concordancia que corrige el azar y que, siempre que sea posible, es preferible a los porcentajes simples de acuerdo (Banerjee, Capozzoli, McSweeney y Sinha, 1999; Cantor, 1996). La fórmula utilizada ha sido la siguiente:

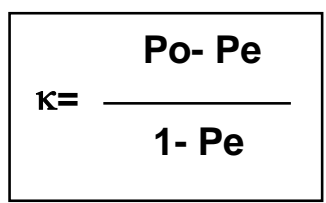

Po es la proporción de concordancia observada realmente (el acuerdo simple entre observadores) y Pe la concordancia observada por azar (sumatorio del producto de frecuencias marginales).

A continuación se muestran los resultados obtenidos en las diferentes pruebas, tomados por binomios, entre cada uno de los observadores independientes (ver tabla 3): 
Tabla 3

Resultados del coeficiente Kappa de Cohen y estadísticos complementarios.

\begin{tabular}{lcccc}
\hline $\begin{array}{l}\text { PARES } \\
\text { OBSERVADORES, } \\
\text { SEGÚN PRUEBA }\end{array}$ & $\begin{array}{c}\text { Valor } \\
\text { Kappa }\end{array}$ & $\begin{array}{c}\text { Error típico } \\
\text { asintótico* }\end{array}$ & $\begin{array}{c}\mathbf{T} \\
\text { aproximada** }\end{array}$ & $\boldsymbol{p}$ \\
\hline CEF 1 VS CEF 2 & 0.60 & 0.051 & 23.47 & .000 \\
CEF 1 VS CEF 3 & 0.70 & 0.048 & 26.55 & .000 \\
CEF 2 VS CEF 3 & 0.47 & 0.053 & 18.43 & .000 \\
CPO 1 VS CPO 2 & 0.57 & 0.058 & 13.13 & .000 \\
CPO 1 VS CPO 3 & 0.70 & 0.053 & 16.14 & .000 \\
CPO 2 VS CPO 3 & 0.53 & 0.058 & 12.36 & .000 \\
PRO 1 VS PRO 2 & 0.70 & 0.051 & 16.39 & .000 \\
PRO 1 VS PRO 3 & 0.81 & 0.043 & 18.71 & .000 \\
PRO 2 VS PRO 3 & 0.57 & 0.056 & 13.52 & .000 \\
CCO 1 VS CCO 2 & 0.73 & 0.047 & 22.37 & .000 \\
CCO 1 VS CCO 3 & 0.79 & 0.043 & 24.06 & .000 \\
CCO 2 VS CCO 3 & 0.65 & 0.050 & 20.46 & .000 \\
\hline
\end{tabular}

* Asumiendo la hipótesis alternativa. Medidas simétricas.

${ }^{* *}$ Empleando el error típico asintótico basado en la hipótesis nula.

Como se desprende de los coeficientes de concordancia mostrados en la tabla anterior, el valor mínimo se sitúa en 0.47 y el máximo en 0.81 , con un predominio de valores superiores a 0.70. Así, globalmente la magnitud de la asociación entre los diferentes binomios (observadores) es alta. Además, todos los errores típicos asintóticos obtenidos están asociados a valores altos de "T" y son estadísticamente significativos ( $p \leq .05)$.

Tomando como base estos resultados, se puede afirmar que la concordancia de los tres observadores ha sido satisfactoria, quedando entonces garantizada la validez de sus valoraciones y resultados.

\section{Resultados}

En la tabla 4 se presentan los promedios de cambios observados en los alumnos participantes en cada una de las cuatro pruebas (CEF, CPO, PRO y CCO). 
Tabla 4

Valores medios obtenidos por los alumnos (europeos y bereberes) en las diferentes pruebas de observación.

\begin{tabular}{lcc}
\hline $\begin{array}{l}\text { PRUEBA } \\
\text { OBSERVACIONAL }\end{array}$ & $\begin{array}{c}\text { Cambios } \\
\text { observados en los } \\
\text { niños Europeos* }\end{array}$ & $\begin{array}{c}\text { Cambios } \\
\text { observados en los } \\
\text { niños Bereberes* }\end{array}$ \\
\hline Cambio expresión facial & 21.36 & 17.59 \\
Cambios posturales & 2.91 & 2.43 \\
Proxemia & 5.25 & 1.65 \\
Contactos corporales & 5.25 & 3.57 \\
\hline
\end{tabular}

${ }^{*}$ Cantidad resultante de promediar las opiniones de los tres observadores.

Como puede observarse, los niños europeos han alcanzado puntuaciones medias más altas en las cuatro pruebas de observación. Así, son los niños de origen cultural europeo los que han obtenido mayores cambios de expresión facial (21.36 frente a 17.56); postural (2.91 frente a 2.43 ); de proximidad (5.25 frente a 1.65); y de contactos corporales (5.25 frente a 3.57).

Con el fin de determinar posibles diferencias estadísticamente significativas entre los dos grupos de niños, según su etnia (europea y bereber), se realizó una prueba de contraste de hipótesis atendiendo a la naturaleza de la escala de medición que se ha aplicado a las variables en cuestión. En este caso, la prueba $t$, verificando los preceptivos supuestos de homoscedasticidad, independencia y normalidad.

Tabla 5.

Valores de $\boldsymbol{t}$ de Student y significación estadística asociada a los mismos.

\begin{tabular}{lcccc}
\hline $\begin{array}{l}\text { PRUEBA } \\
\text { OBSERVACIONAL }\end{array}$ & $\begin{array}{c}\text { Puntuación media } \\
\text { de cambios } \\
\text { observados en los }^{\text {niños europeos }}\end{array}$ & $\begin{array}{c}\text { Puntuación media de } \\
\text { cambios observados } \\
\text { en los niños } \\
\text { bereberes }\end{array}$ & $\boldsymbol{t}$ & $\boldsymbol{p}$ \\
\hline CEF & 21.36 & 17.59 & 3.84 & $.000^{*}$ \\
CPO & 2.91 & 2.43 & -.30 & .760 \\
PRO & 5.25 & 1.65 & 2.99 & $.003^{*}$ \\
CCO & 5.25 & 3.57 & 2.29 & $.024^{*}$ \\
\hline
\end{tabular}

* Diferencias estadísticamente significativas asumiendo un $\alpha=.05$ bilateral. 
Como se desprende de los resultados mostrados en la tabla 5 , se encuentran diferencias estadísticamente significativas en las puntuaciones medias $(p \leq .05)$ entre niños europeos y bereberes, en relación con el conjunto de pruebas analizadas, excepto en la prueba de observación CPO (cambios posturales ante un estímulo). En definitiva, con la excepción de la prueba CPO, en todos los casos los alumnos de origen cultural europeo obtienen puntuaciones medias mayores y estadísticamente significativas $(p \leq .05)$ que los niños bereberes.

Estos resultados confirman que la variable procedencia étnica (europea vs bereber) es determinante en la forma en que los niños objeto de esta investigación se han comportado en relación con una serie de aspectos relacionados con la comunicación no verbal-expresivo corporal: cambios de expresión facial y corporal ante un estímulo, proximidad y contacto corporal.

A continuación se sintetizan los resultados encontrados (ver figura 2), mostrando gráficamente las diferencias comentadas entre los sujetos de ambos grupos:

\section{COMPARATIVA}

PROMEDIOS

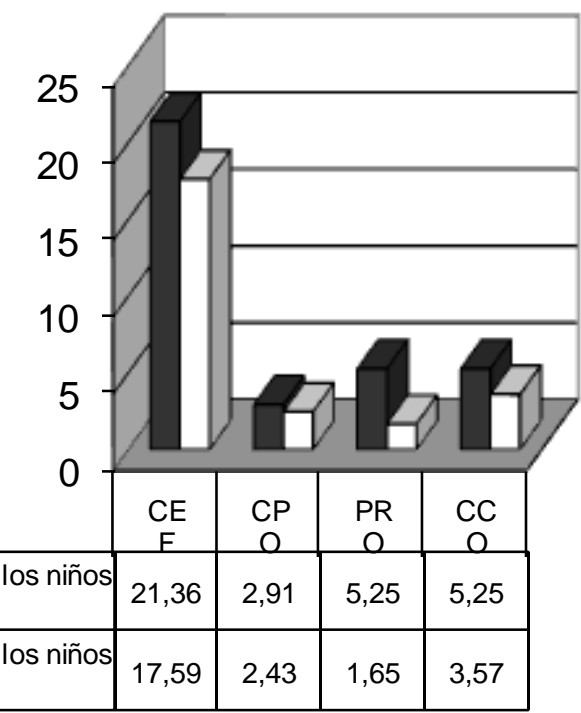

PRUEBAS

Figura 2. Valores promedio comparativos en las pruebas observacionales entre niños de etnia europea y bereber. 


\section{Conclusiones}

El principal hallazgo que se deriva de los resultados obtenidos en este estudio es que se confirman las dos hipótesis propuestas, encontrando que efectivamente existen diferencias estadísticamente significativas entre el comportamiento comunicativo no verbal-corporal que muestran los niños con cultura de origen europeo y los de procedencia cultural bereber en situaciones escolares y en relación con determinados aspectos comunicativos corporales: la expresión facial, el contacto personal y la proxemia, no resultando estadísticamente significativos los cambios posturales.

A pesar de convivir en la misma ciudad norteafricana (Melilla) y asistir a colegios públicos tutelados por un sistema educativo fuertemente arraigado en la cultura española, las diferencias existentes muestran al grupo de alumnos de etnia europea más comunicativo corporalmente que el de origen bereber, lo cual se explica, seguramente, por la continuidad de las costumbres de comportamiento que perviven entre los estudiantes bereberes por encima de su escolaridad en un marco institucional de corte europeo. Indudablemente, en este hecho juega un importante papel la influencia extraescolar que en las costumbres de los alumnos desempeñan todavía sus familias, lo cual guarda relación con el tipo de condiciones generales que actualmente caracteriza la cultura bereber, pues en su gran mayoría los individuos de esta etnia pertenecen a la población norteafricana-magrebí con menor desarrollo económico y educativo (Ennaji, 2005).

Esta situación de desventaja de la cultura bereber está suponiendo, paradójicamente para ella, un factor de preservación de sus costumbres en las expresiones no verbales-corporales; un resistente "impermeable" a la virtual penetración en su identidad cultural de las costumbres del resto de la población europea, que es la mayoría en la ciudad de Melilla. No obstante, este comportamiento de independencia y diferenciación cultural de la cultura amazigh parece que, en términos generales, ha sido una constante en la historia reciente de este pueblo bereber, incluso respecto a países con los que se le supone una mayor cercanía cultural, como Marruecos (McMurray, 2001).

Las implicaciones educativas de estos hallazgos resultan importantes para el buen devenir del proceso de enseñanza-aprendizaje en los entornos escolares donde convivan grupos de alumnos con características interculturales similares a las de este trabajo. 
Si bien parece claro, que el intercambio de comunicación que se establece entre profesores y alumnos es habitualmente asimétrico, el profesorado no debe olvidar que el objetivo de que el alumno aprenda y se obtenga de éste el máximo rendimiento académico queda supeditado al conocimiento por parte del docente de las características de comportamiento y comunicación no verbal-corporal de sus estudiantes (Álvarez, 2003; Formet, 1997), pues el profesor no debe centrarse exclusivamente en transmitir su materia, sino en que el alumno aprenda de forma significativa. Así, el docente debe estar preparado para comprender ciertas conductas y actitudes no verbales que algunos alumnos de etnia diferente a la europea-occidental desarrollarán con seguridad en sus clases diarias (Hall y Hall, 1990; Montepare, 2007).

Esta necesidad para el profesorado de contar con un conocimiento suficiente sobre el repertorio de costumbres de comportamiento corporal y expresión no verbal diferente en el alumnado de cultura amazigh justifica por sí sola la planificación, diseño e implementación de actuaciones curriculares diferenciales en las asignaturas que cursan los estudiantes de educación primaria, especialmente en algunas materias con un gran componente corporal, como la educación física. En este sentido, nos parecen relevantes experiencias recientes sobre diseño curricular en contextos interculturales llevadas a cabo en España durante estos últimos años. Destacamos, por ejemplo, el desarrollo de competencias interculturales, evaluaciones de diagnóstico del alumnado inmigrante, diagnóstico e intervención para el desarrollo de competencias interculturales...

En este contexto, además, adquiere una importancia considerable el concepto de currículum infusionado. En esencia los programas de enseñanza infusionada responden de manera coherente a la problemática intercultural, desarrollando habilidades de pensamiento en conjunto con los contenidos curriculares. A diferencia de otras propuestas, la enseñanza infusionada permite a los alumnos, independientemente del origen étnico de procedencia, aprender a ser estratégicos, ya que relaciona los contextos específicos con determinadas habilidades de pensamiento, por lo que se aprenden estas habilidades apoyadas en el uso práctico y real que tienen (Essomba, 2005, 2007, 2008; Resnick,1999; Salmerón y Rodríguez, 2003). 
Es aquí, por tanto, donde el docente puede lograr su mejor desempeño como formador en un contexto educativo intercultural. Así pues, el docente debe convertirse en una guía y apoyo del aprendizaje del alumno, llegando a ser un comunicador eficaz del conocimiento, de las actitudes y valores necesarios para alcanzar una deseable empatía, en nuestro caso, con los elementos de expresión no verbal-corporal característicos de los estudiantes no autóctonos, por ejemplo, mediante el respeto a patrones gestuales distintivos. De este modo, la escuela debiera formar a sus estudiantes no sólo en adquisición de conceptos, si no también en hábitos de igualdad y responsabilidad entre ellos y respecto a sus profesores.

\section{REFERENCIAS}

Álvarez de Arcaya Ajuria, Helena. (2003). La comunicación no verbal. Interrelaciones entre las expresiones faciales innatas y las aprendidas. Gazeta de Antropología 19. Recuperado el 28 de septiembre de 2009, de: http://www.ugr.es/ pwlac/G19 19Helena AlvarezDeArcaya Ajuria.html

Atkinson, Paul; Hammersley, Martyn. (1998). Ethnography and participant observation. In N. K. Denzin \& Y. S. Lincoln (Eds.), Strategies of qualitative inquiry (pp. 110-136). Thousand Oaks, CA: Sage Publications.

Banerjee, Mousumi; Capozzoli, Michelle; McSweeney, Laura; Sinha, Debajyoti. (1999). Beyond kappa: a review of interrater agreement measures. The Canadian Journal of Statistics, 27(1), 3-23.

Cantor, Alan B. (1996). Sample-Size Calculations for Cohen's Kappa. Psychological Methods, 1(2), 150-153.

Capper, Simon. (2000). Nonverbal Communication and the Second Language Learner: Some pedagogic considerations. JALT Journal. Recuperado el 26 de septiembre de 2008, de: http://www.jalt-publications.org/ttt/articles/2000/05/capper

Eibl-Eibesfeldt, Irenaus. (1970). Ethology. The Biology of Behavior. New York: Holt, Rinehart and Winston, Inc.

Ekman, Paul; Friesen, Wallace V. (1969). Non Verbal behavior in psychotherapy research. Washington: American Psychological Association.

Elfenbein, Hillary Anger; Ambady, Nalini. (2002). On the Universality and Cultural Specificity of Emotion Recognition: A Meta-Analysis. Psychological Bulletin, 128(2), 203-235.

Ennaji, Moha. (2005). Multilingualism, Cultural Identity, and Education in Morocco. New York: Springer. 
Essomba, Miguel Ángel (2005). Liderar escuelas interculturales e inclusivas. Barcelona: Graó.

Essomba, Miguel Ángel (2007). Construir la escuela intercultural: Reflexiones y propuestas para trabajar la diversidad étnica y cultural. Barcelona: Graó.

Essomba, Miguel Ángel (2008). La gestión de la diversidad cultural en la escuela. Barcelona: Graó.

Formet Fernández, María del Mar. (1997). La verbalización de la gestualidad en el aprendizaje de E/LE. Frecuencia-L, 4, 27-31.

Guo, Xiaotin. (1999). Touch. Talking About Non-Verbal Communication: A Corpus Study. American Language Review, 3(3), 27-29.

Hall, Edward Twitchell; Hall, Mildred Reed. (1990). Understanding Cultural Differences. Yarmouth, Maine: Intercultural Press.

Hendry, Joy. (1999). An Introduction to Social Anthropology: Other People's Worlds. Houndmills: Macmillan.

Long, Toni. (1998). A Non-Verbal Communication Teaching Tip. Paper presented at the Annual Meeting of the Eastern Communication Association (89th, Saratoga Springs, NY, April 23-26).

López-Guzmán Guzmán, Tomás; González Fernández, Virgilio; Herrera Torres, Lucía; Lorenzo Quiles, Oswaldo. (2007). Melilla: ciudad fronteriza internacional e intercontinental. Análisis histórico, económico y educativo. Frontera Norte, 37(19), 733.

Marsh, Abigail A.; Adams, Reginal B.; Kleck, Robert E. (2005). Why do fear and anger look the way they do? Form and social function in facial expressions. Personality and Social Psychology Bulletin, 31, 73-86.

Marsh, Abigail A.; Elfenbein, Hillary Anger; Ambady, Nalini. (2003). Nonverbal "accents": Cultural Differences in Facial Expressions of Emotion. Psychological Science, 14(4), 373-376.

McMurray, David A. (2001). In and Out of Morocco. Minneapolis: University of Minnesota Press.

Monaghan, Leila; Goodman, Jane E. (2007). A Cultural Approach to Interpersonal Communication: Essential Readings. Malden, MA: Blackwell Publishers.

Montepare, Joann M. (2007). Nonverbal Behavior in a Global Context: A Time for Dialogue. Journal of Nonverbal Behavior, 31(3), 153-154.

Nichol, Jonh; Watson, Kate. (2000). Videotutoring, Non-Verbal Communication and Initial Teacher Training. British Journal of Educational Technology, 31(2), 135-144. 
Potoker, Elaine. (1993). Management and Training across Cultures: Importance of NonVerbal Communication Strategies-A Case Study. Paper presented at the Annual Conference on Language and Communication for World Business and the Professions (12th, Ypsilanti, MI, April 1-3).

Resnick, Lauren (1999). La educación y el aprendizaje del pensamiento. Buenos Aires: Ed. Aique.

Salmerón, Honorio y Rodríguez, Luis (2003). Desarrollo de estrategias de aprendizaje en educación infantil. Revista de Educación de la Universidad de Granada, 16, 121 143.

Shiraev, Eric B; Levy, David A. (2006). Cross-Cultural Psychology: Critical Thinking and Contemporary Applications. Boston: Pearson.

Vick, Sarah-Jane; Waller, Bridget M; Parr, Lisa A; Smith, Marcia C; Bard, Kim A. (2007). A Cross-species Comparison of Facial Morphology and Movement in Humans and Chimpanzees Using the Facial Action Coding System (FACS). Journal of Nonverbal Behavior, 31(1), 1-20.

Warren, Neil. (1980). Studies in Cross-Cultural Psychology. New York: Academic Press.

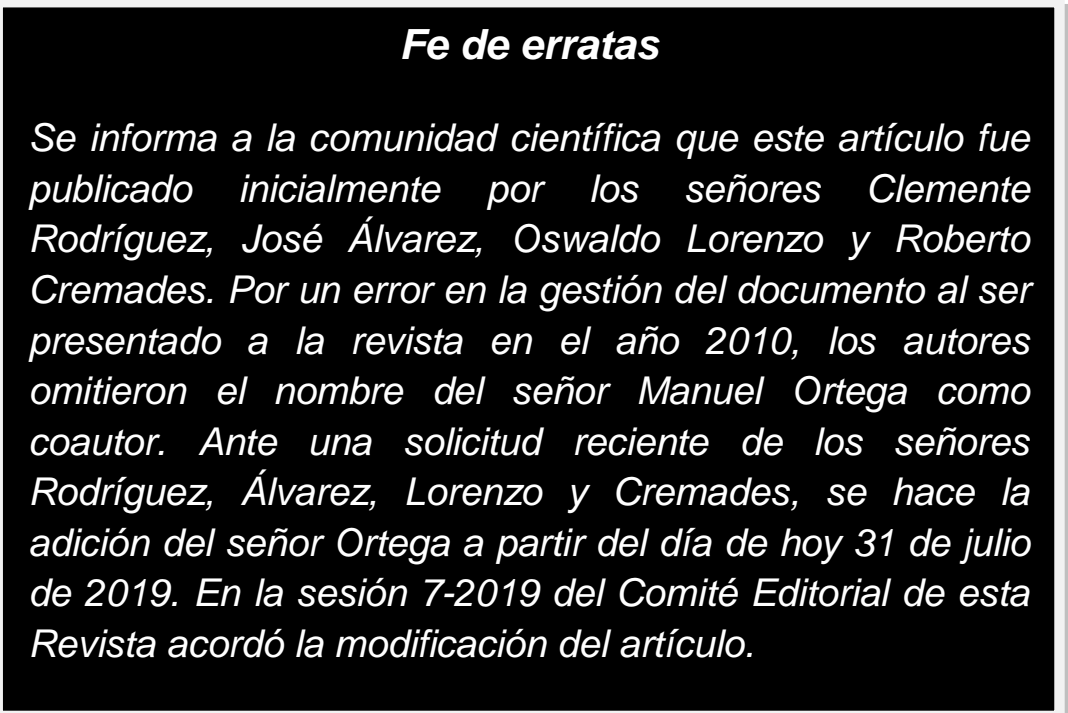

